\title{
Energy Saving Optimization of High Speed Train Based on Speed Prediction Control Curve
}

\author{
Jie $\mathrm{LI}^{1}$, Aihong ZHU, Yuqiong DUAN and Jing ZHANG \\ School of Automation and Electrical Engineering, Lanzhou Jiaotong University, \\ Lanzhou, China
}

\begin{abstract}
In order to study the energy-saving operation of high-speed trains, the energy consumption of trains is taken as the goal, and the speed at the transition point of the operating conditions is the optimization variable, an artificial bee colony algorithm is used to optimize the speed curve across the entire line, the purpose is to obtain the first stage optimization speed curve. On this basis, the conditions of the actual running line are fully considered, and the predictive control algorithm is used to optimize the local prediction of the speed, the purpose is to obtain the second stage optimization speed curve. The simulation results show that compared with the energy consumption in the time-saving mode, the energy consumption after the second prediction optimization is reduced by $19.29 \%$. It is verified that the secondary speed curve obtained by the combination of the global artificial bee colony algorithm and the predictive control algorithm has better performance in energy saving effect. This paper can provide good reference value and practical significance for the energy-saving operation of other vehicles.
\end{abstract}

Keywords. Energy-saving optimization, artificial bee colony algorithm, predictive control algorithm, speed-curve, control transition point

\section{Introduction}

After more than 50 years of development, high-speed railway has won the favor of all countries for its technical and economic advantages such as security, speed, environmental protection and energy saving. At present, the high-speed railway operation mileage has exceeded 35,000 kilometers in China [1], and it has become the unique country with the largest construction scale. However, as the scale of operation continues to expand, the speed of operation continues to increase, greatly increasing the energy consumption required for train operation. According to the 2015-2019 Railway Statistics Bulletin, the national railway energy consumption conversion standard coal is 15.6947 million tons, 15.916 million tons, 16.2165 million tons, 16.2421 million tons and 16.347 million tons [1-5].As can be seen, energy consumption has increased year by year since 2015 , and the comprehensive energy consumption of railways in 2019 increased by 653,000 tons compared to 2015 , an increase of $4.2 \%$. Therefore, it is necessary to study the energy consumption during high-speed train operation.

In 1999, Chang used a differential evolution algorithm to optimize train motion by

\footnotetext{
${ }^{1}$ Corresponding Author, Li Jie, Lanzhou, China; E-mail: 2985014695@qq.com
} 
adjusting the fuzzy membership function [6]. And in 2004, the improved differential algorithm improved the quality of the solution in the optimization of the train's energysaving operating speed curve and achieved a better purpose of energy saving optimization [7]. In 2008, Chuang served energy consumption and running time as objective functions to optimize train idle speed by used artificial neural networks [8]. And in the same year, Acikbas combined genetic algorithm and artificial neural network to solve the energy-saving optimization problem of trains, and achieved a favourable energy-saving effect. However, there is a lack of consideration for the change of external temporary conditions in the study [9]. In 2009, Zhuang Huihua used the idea of predictive control theory to predict the energy-saving operating speed curve of the train, and sought to optimize the operation by the operation mode with less energy consumption to achieve the purpose of energy saving [10]. In 2012, Sun achieved the goal of energy saving by improving the particle swarm optimization algorithm to obtain the optimal train control strategy [11]. In 2015, Ren Xiaoyu used optimized particle swarm optimization algorithm to optimize train energy efficiency in two stages of train operation control and operation planning under rolling ramp and time constraints, and achieved good energy saving effect [12]. In 2016, Huang Youneng used particle swarm optimization to achieve two-stage optimization of train running time between single stations and multiple stations to achieve the goal of energy saving [13]. In 2018, Cao Jiafeng used genetic algorithms to perform two-stage optimization of ramps and idling along the entire line to achieve the purpose of energy saving [14]. In 2019, Ma Xiaona used the firefly algorithm to optimize the position of the operating point of the train's energy-saving operation [15].

In summary, the intelligent algorithm has general applicability to the study of train energy-saving operation optimization problems, and also promotes the development of train energy-saving operation optimization research $[16,17]$. However, most of scholars find the operating speed curve with the lowest train energy consumption by optimizing the operating conditions of the train and the location of the conversion point of the train, and only the running time, distance and speed are considered during the optimization The solution obtained is the global optimal solution, without considering the specific line conditions in the running interval. Therefore, this paper uses offline global artificial bee colony algorithm optimization and online line slope local operation predictive control optimization quadratic speed predictive control curve optimization strategy to find more accurate operating condition transition points, so as to obtain better energy saving effects.

\section{Model Establishment of Train Energy Saving Operation Process}

The energy consumption system of the train is mainly composed of the traction braking power consumption system and the auxiliary energy consumption system. Among them, the traction brake power consumption system refers to the traction braking energy consumption of the train during operation, that is, the train operation energy consumption, is the main part of the train energy consumption system, accounting for up to $80 \%$; the auxiliary energy consumption system refers to the train On-board auxiliary equipment consumes energy. Among them, the main equipment of auxiliary equipment includes air conditioning, lighting, equipment cooling, electrical and electronic devices [18]. Therefore, in this paper, by reducing the energy consumption of traction braking, the train can achieve energy-saving operation on the premise of 
meeting the on-time parking and precise parking. The calculation model of train operation energy consumption is shown in Eq. (1).

$$
E=\int_{0}^{S_{0}} F(v) d s+\int_{0}^{S_{1}} B(v) d s
$$

Where $E$ is traction energy consumption; $F(v)$ is traction force; $S_{0}$ is traction operating distance; $B(v)$ is braking force; $S_{i}$ is braking operating distance.

In this paper, the starting point of the research on the optimization of energy-saving operation of high-speed trains is to let the train run safely in the interval with the most energy-efficient operation sequence under the premise of meeting the punctuality and precise parking according to the operation plan. As a result, the high-speed train energy-saving optimization index model shown in Eq. (2) can be established.

$$
\begin{aligned}
& \min E=\sum_{i=1}^{n} \Delta E_{i}=\int_{0}^{S_{0}} F(s) d s+\int_{0}^{S_{1}} B(v) d s \\
& \text { s.t. }\left\{\begin{array}{l}
n=T / \Delta t \\
0<v_{i}<V_{\max }, v_{0}=0, v_{n}=0 \\
0<s_{i}<S, s_{0}=0, s_{n}=S \\
0<t_{i}<T, t_{0}=0, t_{n}=T
\end{array}\right.
\end{aligned}
$$

Where $E$ is the energy consumption for train operation; $E_{i}$ is the energy consumption for operation calculated at the ith time step; $T$ is the operation time of the train; $\Delta t$ is the time step ( 0.1 in this article); $V_{\max }$ is the maximum speed limit during the operation of the train; $S$ is the running distance of the train.

The relationship between the resultant force, unit resultant force, acceleration, speed and distance during train operation is shown in Eq. (3) to Eq. (6).

$$
\begin{aligned}
& C=F-W_{0}-W_{j}-B \\
& c=\frac{C \times 10^{3}}{M g} \\
& M_{t}=M(1+\gamma) \\
& a=\frac{C}{M_{h}}=\frac{c g}{1000(1+\gamma)}=\frac{0.00981 c}{1+\gamma}
\end{aligned}
$$

Where $C$ is the total force; $F$ is the traction force of the train; $W_{j}$ is the additional resistance of the train, and its additional resistance includes ramps, curves and tunnels; $W_{0}$ is the basic resistance to the train; $B$ is the braking force of the train; $c$ is the unit force of the unit; $M_{t}$ is the convert quality for trains; $\gamma$ is the rotation coefficient, and its value is 0.06 .

According to the size and direction of the traction force, resistance and braking force during the operation of the EMU, the operating conditions of the EMU can be divided into traction, idling and braking. The calculation iteration formula under different working conditions during train operation is shown in Eq. (7). 


$$
\left\{\begin{array}{l}
v_{i+1}=v_{i}+a_{i} \Delta t \\
s_{i}=\frac{v_{i}+v_{i+1}}{2} \Delta t \\
s_{i+1}=s_{i+1}+s_{i}
\end{array}\right.
$$

Where $v_{i}, v_{i+1}$ is the train speed for step $\mathrm{i}$ and $\mathrm{i}+1 ; a_{i}$ is the train running acceleration, which is positive under traction conditions and negative under idle and braking conditions; $S_{i}, S_{i+1}$ is the EMU running distance for step i and step i+1.

\section{High Speed Train energy Saving Optimization Principle}

In the actual running process of high-speed trains, due to its very complicated running state, in order to facilitate the simulation of the running process of high-speed trains, a variety of traction strategies can be established as needed. That is, on the basis of a certain traction algorithm, combined with the actual line conditions, the operation method of train interval operation is formulated [19].

For the running process of the train, it can be divided into three parts: starting and traction process, intermediate running process and inbound braking process [20]. In the starting and traction phases, the train accelerates at the maximum allowable acceleration, so that the train can reach the target operating speed in the shortest running time; in the intermediate running phase, the train adopts traction or partial traction on the uphill slope, and uses inertia or adjustment Speed braking (there is a long downhill on the line); during the braking phase of the station, the train completes the braking process with maximum acceleration.

\section{The First Time Speed Control Curve Optimization}

For the optimization of train operation energy-saving operation, the essence is to find the position of the working condition transition point that meets the constraints of running time, running distance and running speed, etc., which is the optimization of a multidimensional constraint problem. When the train is actually running, because the number of conditions conversion is uncertain, it can take several conditions conversion sequence to operate [21]. The operating condition sequence is prepared in advance, and an intelligent algorithm is used to optimize the planned operating condition sequence to obtain the operating condition conversion point with the best train energy-saving effect. By comparing the traditional swarm intelligence optimization algorithm, it can be seen that artificial bee colony algorithm (ABC) is compared with genetic algorithm (GA), particle swarm optimization algorithm (PSO) and differential evolution algorithm (DE), which has low algorithm complexity and search ability Strong, robust, less set parameters and higher accuracy [22,23]. Therefore, in this paper, the ABC algorithm is used to optimize the calculation to determine the optimal position and speed of the key transition point of the high-speed train operating conditions under the constraints of running speed, running time and running interval. The optimized primary speed control curve is obtained. 


\subsection{Artificial Bee Colony Algorithm}

The $\mathrm{ABC}$ algorithm divides the artificial bee colony into three types: bee collecting, observation bee and reconnaissance bee by simulating the honey collecting mechanism of actual bees. It uses the observation bee to choose the bee to follow according to the probability, and at the same time uses the detection bee to help the algorithm jump out of the local optimal solution and finally find the optimal solution.

By dancing "swing dance" and other methods, the bee-collecting bee tells the observing bee the fitness value of the honey source. Observe the bee calculates the fitness value of the honey source, determine the probability of each honey source being selected according to the honey source's fitness, select a honey source, and collect honey near it to find other honey sources. Detective bees randomly generate new solutions and replace the abandoned honey sources.

In the process of collecting nectar, bees are collected according to Eq. (8) to perform a neighborhood search, using Eq. (9) to replace the honey source, and using Eq. (10) to calculate their fitness value fitness [22]. Observing bees calculate the following probabilities according to Eq. (10) and Eq. (11) and choose to pick bees to follow, and convert them into bees to conduct neighborhood search. The detection bee searches for new honey sources to replace those that are better than the maximum neighborhood search value.

$$
\begin{aligned}
& v_{i j}=x_{i j}+\alpha\left(x_{i j}-x_{k j}\right), \quad i \neq k \\
& x_{i+1}= \begin{cases}v_{i j}, & f_{v} \leq f_{x} \\
x_{i j}, & f_{v}>f_{x}\end{cases} \\
& \text { fitness }_{i}= \begin{cases}\frac{1}{1+f_{i}} & f_{i} \geq 0 \\
\left|f_{i}\right| & f_{i}<0\end{cases} \\
& p_{i}=\frac{\text { fitness }_{i}}{\sum_{i} \text { fitness }_{i}}
\end{aligned}
$$

Where $x_{i j}$ is the jth position of the ith solution; $v_{i j}$ is the new honey source location; $\alpha$ is the domain search coefficient, a random number from 0 to $1 ; f_{v}, f_{x}$ are the fitness values of $v_{i}$ and $x_{i} ; f_{i}$ is the function value; fitness $s_{i}$ is function fitness value; $p_{i}$ is the following probability.

In order to verify the excellent optimization performance of the $\mathrm{ABC}$ algorithm, the test function shown in Eq. (12) is tested using the ABC algorithm, PSO algorithm and DE algorithm.

$$
f(x)=\sum_{i=1}^{d} x_{i}^{2}, x_{i} \in[-100,100]
$$

Where $d$ is the variable dimension, and is taken 100 .

The three-dimensional diagram of the test function shown in Eq. (12) drawn by 
MATLAB is shown in Figure 1. In MATLAB simulation verification, the parameter settings of the artificial bee colony algorithm are shown in Table 1 . The results of simulation and optimization of the test function are shown in Table 2. The average value is the average value obtained by running the optimization program 10 times. Level. The convergence is defined as: (worst value-optimal value)/average value, which indicates the level of convergence performance.The curve of test function optimization convergence is shown in Figure 2.

Table 1. ABC algorithm, PSO algorithm and DE algorithm parameter settings

\begin{tabular}{lll}
\hline ABC Algorithm & PSO Algorithm & DE Algorithm \\
\hline Dimension:100 & Dimension: 100 & Dimension: 100 \\
Group size: 100 & Group size: 100 & Group size: 100 \\
Number of bees:50 & Learning factor: $c_{l}=c_{2}=1.5$ & Initial mutation operator: $F=0.6$ \\
Observe the number of bees:50 & Maximum speed: $v_{\max }=1$ & Crossover operator: $C R=0.5$ \\
Maximum number of searches: & Minimum speed: & Threshold: \\
Limit $=100$ & $v_{\min }=-1$ & $y z=10^{-6}$ \\
The maximum number of & The maximum number of & Maximum evolutionary \\
iterations: max $C y c l e=2000$ & iterations: $T=2000$ & algebra: $G=2000$ \\
\hline
\end{tabular}



Figure 1. Three dimensional graph of test function

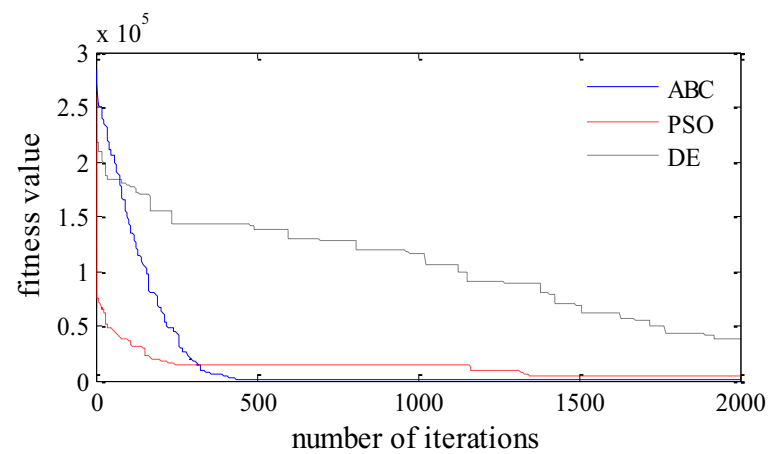

Figure 2. ABC algorithm, PSO algorithm and DE algorithm fitness curve 
Table 2. Sphere function optimization results

\begin{tabular}{llllll}
\hline Function & Algorithm & The optimal value & Worst value & Average value & Convergence \\
\hline \multirow{3}{*}{ Sphere } & ABC & $6.00261 \mathrm{e}-17$ & $2.78079 \mathrm{e}-16$ & $2.64385 \mathrm{e}-16$ & $8.24755 \mathrm{e}-01$ \\
& PSO & $9.76091 \mathrm{e}-15$ & $1.90171 \mathrm{e}-14$ & $9.52552 \mathrm{e}-15$ & $9.71725 \mathrm{e}-01$ \\
& DE & $2.32334 \mathrm{e}-08$ & $7.62161 \mathrm{e}-07$ & $1.91699 \mathrm{e}-07$ & $3.85462 \mathrm{e}+00$ \\
\hline
\end{tabular}

It can be seen from Figure 2 and Table 2 that the ABC algorithm, PSO algorithm and DE algorithm corresponding to the optimal value of the Sphere function have different iteration times, and the precision of the optimal value is different. And when PSO algorithm and DE algorithm calculate function, premature phenomenon often occurs, and the convergence is weak. Comparison shows that the $\mathrm{ABC}$ algorithm is superior to the PSO algorithm and DE algorithm.

\subsection{Optimization Steps of Artificial Bee Colony Algorithm}

The $\mathrm{ABC}$ algorithm is used to optimize the energy-saving operation speed curve of high-speed trains. The objective function is the train energy consumption, and the optimization variable is the speed value at the transition point of the train operating conditions. The specific optimization process is shown in Figure 3. According to the optimization steps in Figure 3, the honey source position corresponding to the minimum fitness value is obtained, that is, the speed value at a set of operating condition transition points corresponding to the lowest train running energy consumption, and the optimized primary speed control curve.

\section{Optimization of Quadratic Speed Predictive Control Curve}

For the energy saving problem of high-speed trains, the simplest and easiest way is to control the train speed. Because the optimization of the primary speed curve is a set of operating condition conversion values with the lowest train running energy consumption found in the whole line under the conditions of running time, running distance and running speed. However, when the train is running on different lines, the driver uses different operation sequences, the resulting train energy consumption is also different, resulting in a difference of up to $30 \%$. Therefore, there is still room for local optimization on specific lines, and the train can achieve energy-saving operation by predicting the energy-saving operation speed of the train. The predictive control algorithm has the characteristics of good control effect and strong robustness, and has a wide range of applications. It is mainly used in processes that do not require accurate mathematical models [10]. Therefore, this paper uses predictive control algorithm, on the basis of the solved primary speed curve, make full use of the line situation, optimize the speed locally, establish the speed prediction model, the rolling optimization of the speed prediction model, and the feedback correction of the speed prediction model to determine More suitable working condition conversion point position, and online real-time adjustment to correct the deviation of the operating speed, to obtain the final speed prediction control curve after the second optimization. 


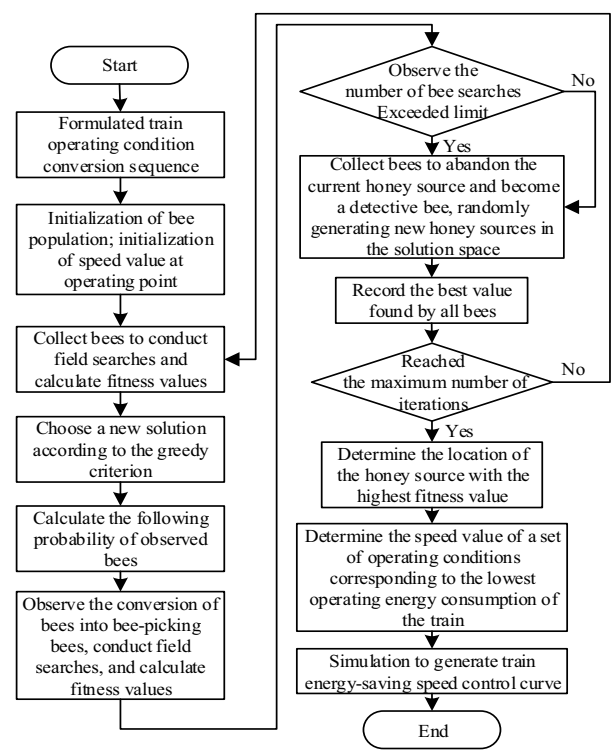

Figure 3. Optimization of the speed curve of high speed train energy saving operation

\subsection{Establishment of Predictive Control Algorithm}

Predictive control is to predict the future state or output based on the object's historical information and hypothetical future input[24]. In this paper, the idea of predictive control is used to predict and optimize the energy-saving speed curve of high-speed trains, and the speed prediction model is established with the speed of the train as shown in Equation 13 to Equation 16.

$$
\begin{aligned}
& \Delta t=\frac{v_{i}-v_{i-1}}{a} \\
& t=\sum \Delta t \\
& \Delta s=\frac{v_{i}^{2}-v_{i-1}^{2}}{2 a} \\
& s=\sum \Delta s
\end{aligned}
$$

Where $v_{i}$ is the final speed of the speed interval; $v_{i-1}$ is the initial speed of the speed interval; $a$ is the acceleration.

\subsection{Predictive Control Algorithm Optimization Steps}

On the basis of the speed curve obtained after the primary optimization of the ABC algorithm, combined with the line data, the predictive control algorithm is used to optimize the energy saving secondary speed curve of the high-speed train. 
Based on the optimized speed curve once, the running speed is partially optimized again to correct the running speed deviation in real time to obtain the optimized secondary speed curve.

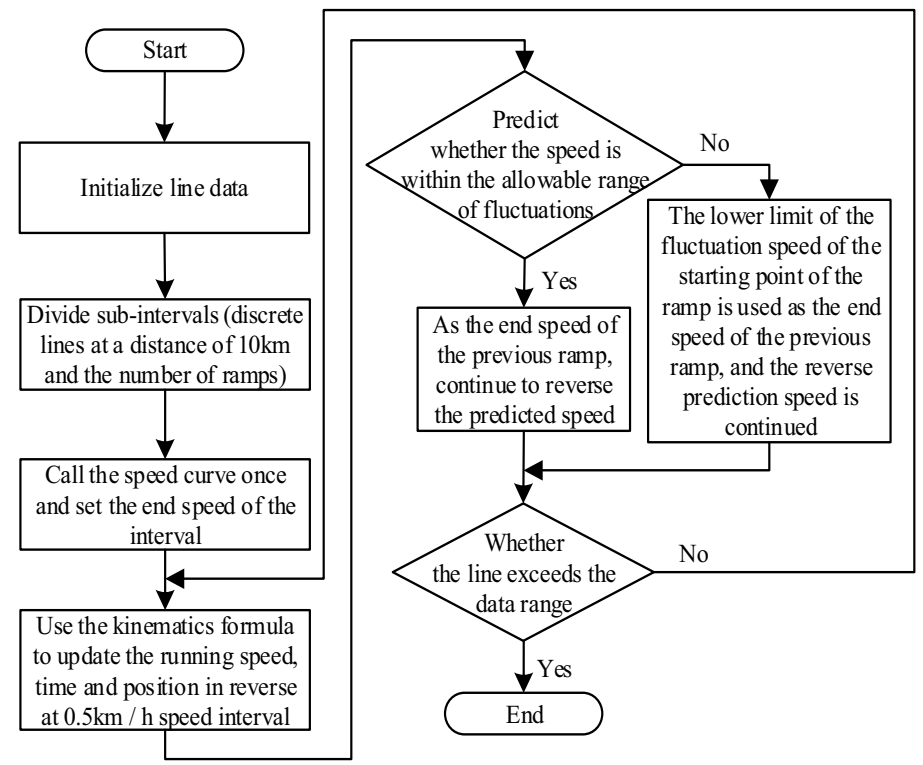

Figure 4. Optimization of secondary speed curve for high speed train energy saving operation

\section{Forecast Results and Analysis}

In order to verify the effectiveness of the method proposed in this paper, this paper takes CRH2 EMU as the experimental simulation object, and the main parameters of the train are shown in Table 3. Taking the Shizhu County Railway Station to the Fengdu Station (k1480.863 - k1529.620) on the Yuli Passenger Line as the simulation line, the interval length is $48.757 \mathrm{~km}$, the line speed limit is $200 \mathrm{~km} / \mathrm{h}$, the scheduled operation time is $1200 \mathrm{~s}$, and the allowed time is $30 \mathrm{~s}$. The longitudinal section is shown in Figure 5.

Table 3. Main train parameters

\begin{tabular}{ll}
\hline Parameter name & Parameter characteristics \\
\hline Full load weight/t & 408.5 \\
Number of people /person & 610 \\
Maximum operating speed/ km/h & 250 \\
Unit basic resistance N/kN & $w_{0}=0.88+0.00744 \times v+0.000114 \times v^{2}$ \\
Additional resistance per ramp N/kN & $w_{\mathrm{i}}=i$ \\
Traction characteristics $/ \mathrm{kN}$ & $F=176-0.36 \times v \quad 0 \leq v \leq 125 \mathrm{~km} / \mathrm{h}$ \\
& $F=16250 / v \quad v>125 \mathrm{~km} / \mathrm{h}$ \\
Regenerative braking characteristics $/ \mathrm{kN}$ & $B=172 \quad 15 \mathrm{~km} / \mathrm{h} \leq v \leq 70 \mathrm{~km} / \mathrm{h}$ \\
& $B=187-0.225 \times v \quad v>70 \mathrm{~km} / \mathrm{h}$ \\
\hline
\end{tabular}


Shizhu County

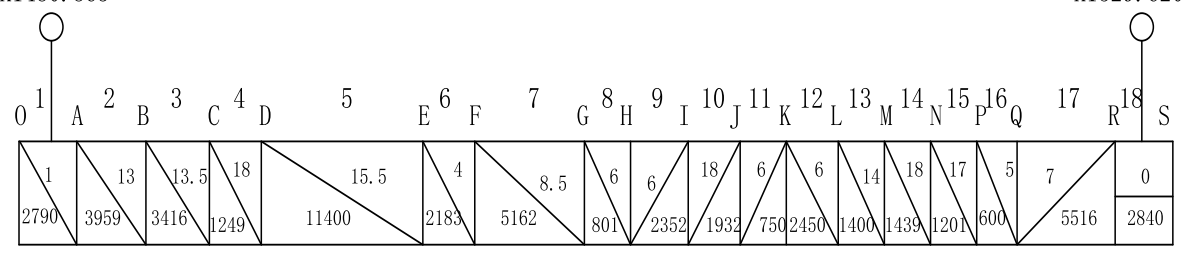

Figure 5. Longitudinal section of the line

According to the traction force, resistance and braking force characteristics of CRH2 EMUs, the corresponding train traction, resistance and braking characteristic curves can be simulated as shown in Figure 6 .

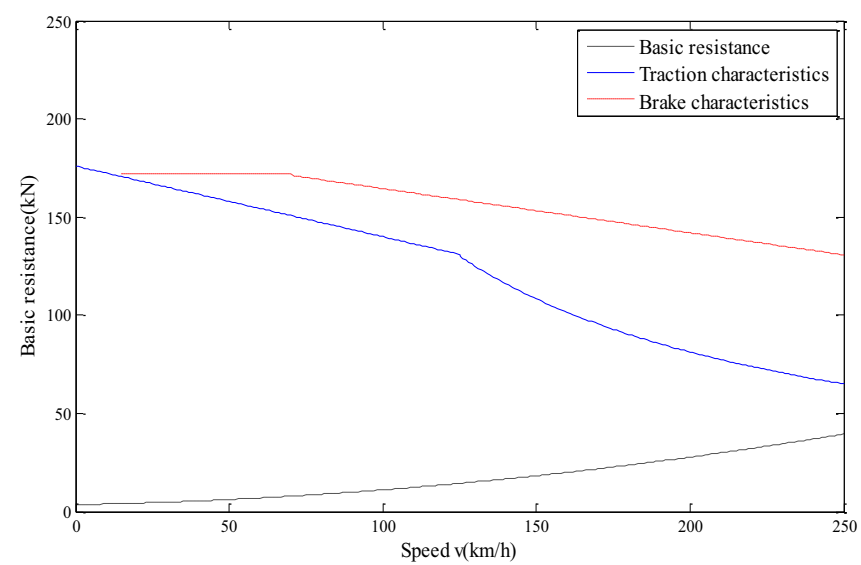

Figure 6. $\mathrm{CRH} 2$ traction, drag and braking characteristics

Combining the above theoretical knowledge, the simulation obtained the running speed curve of the train before optimization and after the optimization of the energy saving strategy of the line ramp as shown in Figure 7.

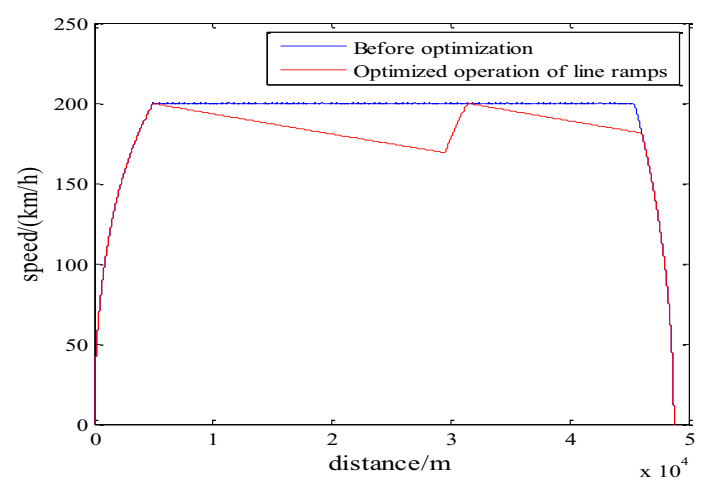

Figure 7. Speed curve before optimization and after optimization of line ramp operation 
The ABC algorithm is used throughout the entire line to optimize the calculation and determine the optimal position and speed of the key transition point of the highspeed train operating conditions under the constraints of operating speed, operating time and operating interval. In this paper, the operation strategy of "traction-lazy traveltraction-lazy travel-braking" is adopted in the whole section of the train. The calculated primary speed control curve optimized by the ABC algorithm is shown in Figure 8. On the basis of global optimization, combined with line conditions, using predictive control algorithm ideas online local optimization to obtain the optimized secondary speed curve is shown in Figure 9, the performance indicators before and after optimization are shown in Table 4.

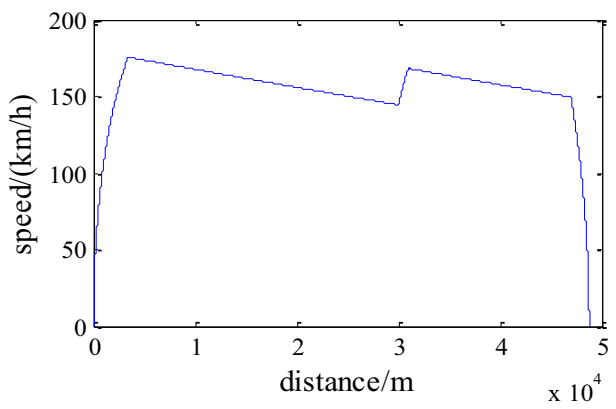

Figure 8. The speed curve after optimization by $\mathrm{ABC}$ algorithm

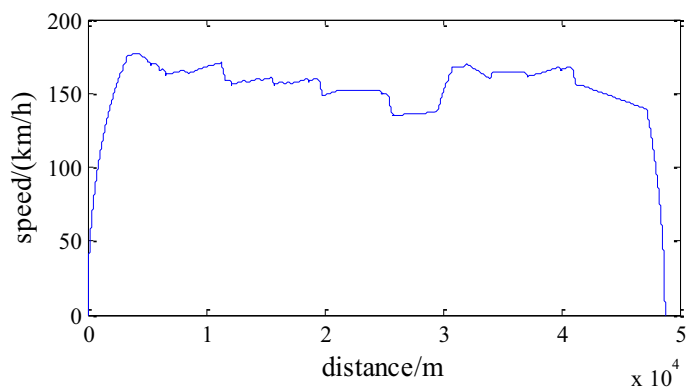

Figure 9. The quadratic prediction velocity curve optimized by predictive control algorithm

Table 4. Performance indicators before and after optimization

\begin{tabular}{lllll}
\hline Manipulation method & $\begin{array}{l}\text { operation } \\
\text { hours } / \mathbf{s}\end{array}$ & $\begin{array}{l}\text { Running } \\
\text { distance } / \mathbf{k m}\end{array}$ & $\begin{array}{l}\text { Total energy } \\
\text { consumption /J }\end{array}$ & $\begin{array}{l}\text { Energy saving } \\
\text { ratio /\% }\end{array}$ \\
\hline $\begin{array}{l}\text { Before optimization } \\
\begin{array}{l}\text { Optimized operation of } \\
\text { line ramps }\end{array}\end{array}$ & 1035.69 & 48.75719 & $1.902 \times 10^{9}$ & - \\
$\begin{array}{l}\text { One speed curve optimization } \\
\text { Forecast and optimization of } \\
\text { quadratic speed curve }\end{array}$ & 1053.52 & 48.75727 & $1.799 \times 10^{9}$ & -5.40 \\
\hline
\end{tabular}

By comparing the performance indicators obtained by the above several maneuvering strategies, it can be seen that due to the continuous traction-lazy running conditions before the optimization of the train, the train runs close to the speed limit, 
resulting in excessive energy consumption of the train, which is $1.902 \times 10^{9} \mathrm{~J}$ The shortest running time is $1035.69 \mathrm{~s}$. Combining with the train energy-saving optimization principle, the simulation shows that the energy consumption of the line slope operation optimization is $1.799 \times 10^{9} \mathrm{~J}$, which is a reduction of $5.40 \%$ compared with the operation mode before optimization. The energy consumption obtained by the second optimization method of artificial bee colony algorithm and predictive control algorithm mentioned in this paper is $1.535 \times 10^{9} \mathrm{~J}$. Compared with the operation strategy before optimization, the energy consumption is saved by $19.29 \%$, and the energy consumption is significantly reduced. (In the morning and evening operation time within $30 \mathrm{~s}$ ), precise parking (meeting the technical requirements of $0.3 \mathrm{~m}$ parking error) requirements, and can well guide the driver to operate the train, thus verifying the global artificial bee colony algorithm and The effectiveness of predictive optimization algorithm combined with predictive control algorithm in train energy saving.

\section{Conclusion}

This article uses offline global artificial bee colony algorithm and online prediction control of local operation on ramps to optimize train running curve, the result can not only solve the problem of high energy consumption of train operation, but also can be adjusted online in real time to correct the deviation of the running speed. The driver can adjust the deviation of the running speed online in real time according to the unexpected situation that may occur in actual operation to realize the real-time speed control of the train through the optimized speed curve.

In order to solve the problem of optimizing the energy-saving operation of trains, the quadratic optimization algorithm mentioned is used to predict and adjust the running speed on the basis of the optimized energy-saving operation conditions to achieve the purpose of saving energy in this paper. Taking the CRH2 single-point highspeed train and Yuli passenger line as experimental simulation objects, The optimized performance indexes of the train obtained from the simulation justified, the train can be verified on the premise of ensuring safe, accurate parking and on-time operation. The consumption is reduced by $19.29 \%$, and achieved a better energy saving effect.

For the energy-saving control of trains, the key point of research is how to determine the train operating condition sequence. In this paper, a set of train operating condition sequences are formulated based on the selected actual line conditions. The simulation results show that the method proposed in this article can reduce the effect of train operation energy consumption, and the proposed operating condition sequence is reasonable and correct.

\section{Acknowledgement}

Fund Project: National Natural Science Foundation of China (61661027)

\section{References}

[1] National Railway Administration. 2018 Railway Statistics Bulletin. Beijing: National Railway Administration, 2019. 
[2] National Railway Administration. 2015 Railway Statistics Bulletin. Beijing: National Railway Administration, 2016.

[3] National Railway Administration. 2016 Railway Statistics Bulletin. Beijing: National Railway Administration, 2017.

[4] National Railway Administration. 2017 Railway Statistics Bulletin. Beijing: National Railway Administration, 2018.

[5] National Railway Administration. 2019 Railway Statistics Bulletin. Beijing: National Railway Administration, 2020.

[6] Chang CS, Xu DY, Quek HB. Pareto-optimal set based multi-objective tuning of fuzzy automatic train operation for mass transit system. IEE Proceedings-Electric Power Applications,1999,Vol.146(5)

[7] Chang CS, Kwan CM. Evaluation of evolutionary algorithms for multi-objective train schedule optimization. AI 2004: Advances in Artificial Intelligence. Springer Berlin Heidelberg,2004.

[8] Chuang HJ, Chen CS, Lin CH, et al. Design of optimal coasting speed for saving social cost in mass rapid transit systems. Electric Utility Deregulation and Restructuring and Power Technologies, 2008. DRPT 2008. Third International Conference on. IEEE,2008:2833-2839.

[9] Acikbas S, Soylemez MT. Coasting point optimization for mass rail transit lines using artificial neural networks and genetical gorithms. IET Electric Power Applications,2008,2(3):172-182.

[10] Zhuang HH. Research on locomotive energy-saving operation control system based on predictive control theory. Chengdu: Southwest Jiaotong University, 2009.

[11] Sun SY, Li Y, Xu HY. Energy consumption optimization for high-speed railway based on particle swarm algorithm. Computational Intelligence and Communication Networks (CICN) 2012 Fourth International Conference on. IEEE,2012:879-882.

[12] Ren XY. Research on train energy-saving strategy based on optimized particle swarm optimization. Beijing: Beijing Jiaotong University, 2015.

[13] Huang YN, Gong SF, Cao Y, et al. Optimization model for energy-saving driving of urban rail trains based on particle swarm optimization. Journal of Transportation Engineering, 2016, 16 (02): 118-124 + 142.

[14] Cao JF, Liu B. Simulation study on energy-saving operation of high-speed trains based on 2-stage optimization. Journal of Railway Science and Engineering, 2018, 15 (04): 821-828.

[15] Zhu AH, Ma XN, Duan YQ, Li J, Li J. Energy-saving optimization of high-speed train tracking operation process. Sensors and Microsystems, 2019, 38 (12): 47-49+53.

[16] Farooq A, Xie MW, Stoilova S, Ahmad F, Guo M. Transportation planning through GIS and multicriteria analysis: case study of Beijing and XiongAn. Journal of Advanced Transportation, 2018, 858-875.

[17] Farooq A, Xie MW, Stoilova S, Ahmad F. Multicriteria evaluation of transport plan for high-speed rail: an application to Beijing-Xiongan. Mathematical Problems in Engineering,2019,1136-1159.

[18] Ma WW, Li LQ. EMU energy consumption measurement and evaluation. Changsha: Central South University Press, 2015: 5-6.

[19] Yang PS. Real-time simulation of subway train operation based on PC. Chengdu: Southwest Jiaotong University, 2007.

[20] Song WT, Tan M, Cai WC, et al. Research on energy-saving maneuvering strategies of high-speed trains. Journal of Railway Science and Engineering, 2016, 13 (03): 423-429.

[21] Duan YQ, Zhu AH, Ma XN, et al. Research on optimization of high-speed train operation energy saving based on artificial bee colony algorithm. Railway Standard Design, 2019, 63 (09): 163-168.

[22] Qin QD, Cheng S, Li L, et al. Review of artificial bee colony algorithm research. Journal of Intelligent Systems, 2014, 9 (2): 127-135.

[23] Wang H. A comparative study on the performance of artificial bee colony algorithm. Journal of Hebei Engineering and Technical College, 2015 (01): 41-44.

[24] Fu SG. Research on Model Predictive Control Algorithm of AC Servo System Considering Interference and Friction. Nanjing: Southeast University, 2018. 